The Egyptian Journal of Hospital Medicine (April 2019) Vol. 75 (5), Page 2794-2800

\title{
The SYNTAX Score and Angiographic "No-Reflow" in Patients with Acute Myocardial Infarction Undergoing Percutaneous Coronary Intervention
}

Ahmad Hassan Sadek*, Ezz EL Din AL Sawy, Mohammed Saad Al Gammal, and Mohammad Ahmad Mosaad

Department of Cardiology, Faculty of Medicine, Al-Azhar University, Cairo

*Corresponding author: Ahmad Hassan Sadek, Mobile: (+20)01069724760

\begin{abstract}
Background: in patients with acute myocardial infarction, the immediate therapeutic goal is to establish patency of the infarct-related artery and to achieve optimal myocardial tissue reperfusion. The Synergy between percutaneous coronary intervention with TAXUS and cardiac surgery (SYNTAX) score (SS) quantifies the extent and complexity of angiographic coronary artery disease.

Patients and Methods: a total of 543 patients presenting with acute myocardial infarction undergoing primary PCI for the STEMI patients and early invasive strategy for the NSTEMI patients, both admitted within 24 hours from the symptoms onset, were analyzed. SS, thrombolysis in myocardial infarction (TIMI) flow grade score, and TIMI myocardial blush grade score (MBG) were determined in all patients. No-reflow was considered as the prescence of TIMI blood flow in the infarct related artery (IRA) $\leq 2$ or TIMI grade 3 with myocardial blush grade (MBG) 0 or 1, at least 10 minutes after the end of the PCI procedure.

Results: no-reflow was observed in $26 \%$ of patients. The mean SS of the no-reflow group was higher than that of the TIMI III flow group . On multivariate logistic regression analysis a long target lesion (OR= 8.637, 95\% C.I $1.975-37.768, \mathrm{p}=0.004$ ) were found to be significantly associated with no-reflow and were the independent predictors of no-reflow phenomenon. The cutoff value of SS obtained by the receiver-operator characteristic curve analysis was 31 for the prediction of no-reflow .
\end{abstract}

Conclusion: the SS is a predictor of no-reflow in patients with acute myocardial infarction treated with percutaneous coronary intervention.

Keywords: acute myocardial infarction, percutaneous coronary intervention, No-reflow, SYNTAX score

\section{INTRODUCTION}

In patients with acute myocardial infarction, the successful restoration of epicardial culprit coronary artery patencydoes not always guarantee restoration of myocardial tissue-level perfusion \& salvage of myocardium at risk of ischemia ${ }^{(\mathbf{1})}$. In a variable proportion of patients with acute myocardial infarction, however, microcirculatory impairment may persist after epicardial coronary artery recanalization following PCI and may attenuate its beneficial impact $^{(2)}$. The phenomenon of myocardial no-reflow is defined as inadequate myocardial perfusion through a given segment of the coronary circulation without angiographic evidence of mechanical vessel obstruction $^{(3)}$.

Further, recent studies have revealed that distal embolizationof thrombusand/or plaque contents are one of the major causes ofno-reflow. It may be critically important, therefore, to be able to predict which lesions are high risk for myocardial no-reflow prior to beginning percutaneous coronary intervention $(\mathrm{PCI})^{(4)}$.

Accurate detection of 'no-reflow' is thus crucial because it is independently associated with low ventricular ejection fraction, adverse left ventricular remodeling, malignant arrhythmias, cardiac failure, as well as mortality both at short- and long-term followup $^{(5)}$.

The SYNTAX (synergy between percutaneous coronary intervention with TAXUS and cardiac surgery) score has been shown to be predictive of clinical outcome in different clinical settings in patients undergoing percutaneous coronary intervention $(\mathrm{PCI})^{(6)}$.

\section{PATIENTS AND METHODS}

\section{Study population:}

The study was conducted on 543 patients with acute myocardial infarction undergoing primary PCI for the STEMI patientsand early invasive strategy for the NSTEMI patients, both admitted within 24 hours from the symptoms onset to the cath lab. Of Al-Hussein University hospital during the period from 11/2015 to 11/2017.

The study was approved by the Ethics Board of Al-Azhar University and an informed written consent was taken from each participant in the study.

The study inclusion criteria were patients presenting with an evidence of myocardial necrosis in a clinical setting consistent with acute myocardial ischemia. Under these conditions the following criteria meets the diagnosis for myocardial infarction ${ }^{(7)}$ : Detection of a rise and /or fall of cardiac biomarkers values (preferably cardiac troponin (cTn) with at least one of the following:

○ Symptoms of ischemia. 
- New or presumed new significant ST-segment-T wave (ST-T) changes or new left bundle branch block(LBBB).

- Development of pathological Q waves in the ECG.

- Imaging evidence of new loss of viable myocardium or new regional wall motion abnormality.

- Identification ofan intracoronary thrombus by angiography.

\section{Acute myocardial infarction patients included:}

1-STEMI (ST segment elevation myocardial infarction).

2- NSTEMI (non- ST segment elevation myocardial infarction).

The exclusion criteria were patients with a prior CABG surgery, patients with prior PCI and stenting and patients with poor quality coronary angiograms.

All enrolled patients underwent the following: Full history taking.

Clinical examination, 12 lead electrocardiography, serial cardiac biomarkers, renal functions, weight and height measurements, Echo-cardiographic study at the hospital stay.

A written informed consent was obtained from all the patients enrolled in the study and was approved from the local Ethical Committee.

\section{Angiographic procedure}

PCI was done to treat the de novo culprit lesions with significant stenosis in the native coronary artery. The culprit lesion was identified on the basis of E.C.G, transthoracic echocardiography and coronary angiography.theSYNTAX score was calculated according to The SYNTAX score algorithm ${ }^{(8)}$.

Angiographic criterion was used for the diagnosis of no reflow. Coronary angiography was performed according to the standard criteria. Offline analysis of digital angiograms was performed in the core laboratory using automated edge detection.

The initial and postprocedural blood flow in the infarct-related artery was gradedaccording to the Thrombolysis in Myocardial Infarction (TIMI) flow grading system ${ }^{(9)}$ and TIMI myocardial blush grade (MBG) score ${ }^{(10)}$. The diagnosis of no reflow required the following criteria ${ }^{(11)}$ :

- Angiographic evidence of reopening of occluded coronary arteryand successful stent placement with no evidence of flow-limitingresidualstenosis ( $\geq$ $50 \%$ ), dissection, spasm, or apparent thrombus and

- Angiographic documentation of a TIMI flow grade $\leq$ 2or TIMI grade 3 with MBG 0 and 1, atleast 10 minutes after the end of PCI procedure.

Angiographic criterion of $25 \%$ residual stenosis was adopted as a definition of successful PCI and the endpoint of the interventional procedure.

\section{Statistical study:}

All statistical studies were carried out using the statistical package for social sciences (18.0 for Windows; SPSS Inc., Chicago, Illinois, USA).

Quantitative variables were expressed as mean \pm SD. Qualitative data were expressed as counts and percentages. The Student t-test and the $\chi 2$-test were used to compare quantitative and qualitative values, respectively. Multivariable logistic regression analysis was performed to identify independent predictors of no-reflow phenomenon. The receiving operator characteristic (ROC) curve was used to detect optimal cutoff values of Syntax score for predicting noreflow. A P value less than 0.05 was considered statistically significant.

\section{RESULTS}

Five hundred forty three patients with acute myocardial infarction who underwent primary PCI for the STEMI patients (362) and early invasive strategy for the NSTEMI patients (181), both admitted within 24 hours from the symptoms onset were included in the study. Patients were divided into two groups according to the TIMI flow post PCI; the TIMI III flow group (group I) included 402 patients (74\%) and no-reflow group (group II) that included 141 patients (26\%).

\section{Baseline clinical characteristics}

There were no significant differences between the two groups in terms on gender, DM , HTN, Dyslipedimia, smoking status, BMI, the family history of CAD, the hemodynamic profile and the types ofmyocardial infarction.Patients in the Noreflow group were older, had higher prevalence of PVD, higher peak CKMB levels, higher RBS levels on admission, higher level of killip class (III ( IV), and they tended to have more worse LV contractility $(P<0.001,0.018,<0.001,<0.001$, $<0.001$, and $<0.001$, respectively, Table 1$)$. 
Table (1): Comparison between demographic and clinical data in group I \&II

\begin{tabular}{|c|c|c|c|}
\hline 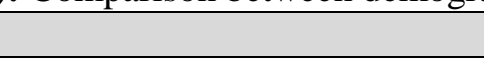 & Group I (reflow) & Group II (no-reflow) & P value \\
\hline Age & $55.19 \pm 10.88$ & $63.19 \pm 9.6$ & $<0.001$ \\
\hline Male gender & $351(87.3 \%)$ & $132(93.6 \%)$ & 0.236 \\
\hline Female gender & $51(12.6 \%)$ & $9(6.3 \%)$ & \\
\hline Smoker & $243(60.4 \%)$ & $89(63.1 \%)$ & 0.213 \\
\hline DM & $162(40.29 \%)$ & $57(40.4 \%)$ & 0.988 \\
\hline HTN & $132(32.8 \%)$ & $48(34 \%)$ & 0.880 \\
\hline Dyslipedimia & $249(61.9 \%)$ & $93(65.9 \%)$ & 0.624 \\
\hline Family history of CAD & $45(11.1 \%)$ & $9(6.3 \%)$ & 0.343 \\
\hline BMI & $27.45 \pm 3.48$ & $28.39 \pm 2.97$ & 0.125 \\
\hline Peripheral vascular disease & $32(8 \%)$ & $41(29.16 \%$ & 0.018 \\
\hline SBP & $127.74 \pm 25.31$ & $129.11 \pm 33.20$ & 0.770 \\
\hline DBP & $78.82 \pm 11.42$ & $78.43 \pm 15.6$ & 0.854 \\
\hline HR & $81 \pm 7.2$ & $76 \pm 10.1$ & 0.683 \\
\hline Killip class & & & \\
\hline I & $246(61.1 \%)$ & $36(25.5 \%)$ & \\
\hline II & $126(31.3 \%)$ & $78(55.3 \%)$ & $<0.001$ \\
\hline III & $27(6.7 \%)$ & $24(17 \%)$ & \\
\hline IV & $3(0.74 \%)$ & $3(2.1 \%)$ & \\
\hline AWMI & $150(37.3 \%)$ & $48(34 \%)$ & 0.836 \\
\hline IWMI & $52(12.9 \%)$ & $24(17 \%)$ & 0.309 \\
\hline IWMI + RVMI & $64(15.9 \%)$ & $20(14.1 \%)$ & 0.862 \\
\hline Lateral STEMI & $2(0.49 \%)$ & $2(1.4 \%)$ & 0.772 \\
\hline NSTEMI & $13433.3 \%)$ & $47(33.28 \%)$ & 0.988 \\
\hline Peak CKMB & $234.71 \pm 136.21$ & $403.00 \pm 144.15$ & $<0.001$ \\
\hline Creatinine Clearance & $96.42 \pm 17.42$ & $93.25 \pm 10.37$ & 0.970 \\
\hline RBS & $144.2 \pm 70.3$ & $275.0 \pm 76.9$ & $<0.001$ \\
\hline Total cholesterol & $217.74 \pm 50.05$ & $219.53 \pm 51.30$ & 0.836 \\
\hline EF & $53.1 \pm 6.0$ & $45.7 \pm 7.2$ & $<0.001$ \\
\hline
\end{tabular}

DM, Diabetes mellitus; HTN, hypertension; CAD ,coronary artery disease; BMI, body mass index; SBP, systolic blood pressure; DBP, diastolic blood pressure; AWMI, anterior wall myocardial infarction; IWMI, inferior wall myocardial infarction; RVMI, right ventricular myocardial infarction; STEMI, ST segment elevation myocardial infarction; RBS, random blood sugar; EF, ejection fraction.

\section{Angiographic \& PCI Data}

- There was a significant difference between patients within both groups for their SYNTAX score denoting higher predicted risks and higher anatomical complexity for No-reflow patients $(22.2 \pm 5.8$ vs $37.4 \pm 3.8$ for TIMI III \& no-reflow patients respectively with a $\mathrm{p}$ value $<0.001$ ).

- STEMI patients who experienced no-reflow had a longer symptom to balloon time $(7.8 \pm 2.4 \mathrm{~h}$ for noreflow vs $3.4 \pm 1.6 \mathrm{~h}$ for TIMI III group with a $\mathrm{p}$ value $<0.001)$ Unlike for the NSTEMI patients who experienced no-reflow who had a median time to revascularization of $19 \pm 2.7 \mathrm{~h}$ which is Comparable to that on TIMI III flow group $16 \pm 1.8 \mathrm{~h}$ with a $\mathrm{p}$ value of 0.074 (table 2).

- There were no significant differences between the two groups regarding the number of diseased vessels, infarct-related artery, the target lesion locations, reference vessel diameter, stent length, stent diameter, rate of use of thrombus aspiration device. No-reflow was more common in patients who had a low $(\leq 1)$ initial TIMI flow ( $\mathrm{p}$ value $<0.001$ ) and a low initial TMP grade $(\leq 1)$ (p value $<0.001$ ), also for patients with high thrombus burden $(\mathrm{P}<0.001)$, and those had longer lesions $>20 \mathrm{~mm}$ ( $\mathrm{P}$ value $<0.001)$ (Table 3). 
Table (2): No-reflow \& acute myocardial infarction (STEMI \& NSTEMI): Symptom to balloon time.

STEMI, ST segment elevation myocardial infarction; NSTEMI, non- ST segment elevation myocardial infarction

\begin{tabular}{|c|c|c|c|}
\hline \multirow{2}{*}{} & \multicolumn{2}{|c|}{$\begin{array}{c}\text { Median symptom to balloon } \\
\text { P- } \\
\text { time }\end{array}$} & \multirow{2}{*}{ Value } \\
\cline { 2 - 3 } & TIMI III & No-reflow & $<0.001$ \\
\hline STEMI & $3.4 \pm 1.6 \mathrm{~h}$ & $7.8 \pm 2.4$ & 0.074 \\
\hline NSTEMI & $16 \pm 1.8$ & $19 \pm 2.7$ & \\
\hline
\end{tabular}

Table (3): Angiographic Data in the TIMI III \& No-reflow groups.

\begin{tabular}{|c|c|c|c|}
\hline & TIMI III & No-reflow & P value \\
\hline IRA & $225(55.9 \%)$ & $72(51.1 \%)$ & \\
\hline LAD & $33(8.3 \%)$ & $9(6.4 \%)$ & \\
\hline LCX & $3(0.7 \%)$ & 0 & \\
\hline Ramus & $144(35.8 \%)$ & $60(42.6 \%)$ & \\
\hline \multicolumn{4}{|l|}{ RCA } \\
\hline \multicolumn{4}{|l|}{ Initial TIMIflow } \\
\hline $0 / 1$ & $189(47 \%)$ & $129(91.5 \%)$ & $<0.001$ \\
\hline $2 / 3$ & $213(53 \%)$ & $12(8.5 \%)$ & $<0.001$ \\
\hline \multicolumn{4}{|l|}{ Initial TMP Grade } \\
\hline $0 / 1$ & $225(56 \%)$ & $138(97.9 \%)$ & $<0.001$ \\
\hline $2 / 3$ & $177(44 \%)$ & $3(2.1 \%)$ & $<0.001$ \\
\hline \multicolumn{4}{|l|}{ Target lesionlocation } \\
\hline Proximal & $216(53.7 \%)$ & $93(66 \%)$ & 0.346 \\
\hline Mid & $162(40.3 \%)$ & $42(29.8 \%)$ & \\
\hline Distal & $24(6 \%)$ & $6(4.3)$ & \\
\hline Total occlusion & $246(61.2 \%)$ & $102(72.3 \%)$ & 0.059 \\
\hline High thrombus burden & $60(15 \%)$ & $93(66 \%)$ & $<0.001$ \\
\hline Reference vessel diameter & $3.204 \pm 0.377$ & $3.123 \pm 0.270$ & 0.341 \\
\hline \multicolumn{4}{|l|}{ Number of diseaded vessels } \\
\hline 1 & $101(25 \%)$ & $28(20 \%)$ & \\
\hline 2 & $153(38 \%)$ & $48(33 \%)$ & \\
\hline$\geq \mathbf{3}$ & $148(37 \%)$ & $64(45 \%)$ & \\
\hline Thrombus aspiration alone & $3(0.74 \%)$ & $3(2.1 \%)$ & 0.436 \\
\hline \multicolumn{3}{|l|}{ Stenting after thrombus } & 0.029 \\
\hline Direct stenting alone & $123(30.5 \%)$ & $18(12.7 \%)$ & 0.016 \\
\hline Pre-dilatation & $159(39.5 \%)$ & $66(46.8 \%)$ & 0.385 \\
\hline Post-dilatation & $123(30.5 \%)$ & $63(44.6 \%)$ & 0.080 \\
\hline \multicolumn{4}{|l|}{ Target lesion length } \\
\hline$>20 \mathrm{~mm}$ & $132(32.8 \%)$ & $114(80.8 \%)$ & $<0.001$ \\
\hline$<20 \mathrm{~mm}$ & $267(66.4 \%)$ & $27(19.1 \%)$ & \\
\hline Stent Length & $3.2 \pm 0.364$ & $3.13 \pm 0.25$ & 0.341 \\
\hline Stent diameter & $22.11 \pm 5.0$ & $20.17 \pm 5.9$ & 0.122 \\
\hline
\end{tabular}

IRA, infarct-related artery; TIMI; thrombolysis in myocardial infarction, TMP; TIMI myocardial perfusion grade

A multivariable logistic regression model was built to identify the independent predictors of no-reflow. The SYNTAX score (odds ratio $=1.833,95 \%$ confidence interval, $1.139-2.951, \mathrm{P}=0.013$ ), the time to reperfusion $>$ 6 hours for STEMI patients ( $\mathrm{OR}=13.844,95 \%$ C.I. $3.214-59.636, \mathrm{P}=0.003)$, age $>60$ years $(\mathrm{OR}=8.884,95 \%$ C.I.2.145 - 36.800, $\mathrm{P}=0.003)$, low initial TIMI flow $(\leq 1)(\mathrm{OR}=20.861,95 \%$ C.I.1.739 $-250.290, \mathrm{p}=0.017$, a long target lesion $(\mathrm{OR}=8.637,95 \%$ C.I1.975 $-37.768, \mathrm{p}=0.004)$ were found to be significantly associated with no-reflow and were the independent predictors of no-reflow phenomenon ((Table 4)). ROC curve analysisidentified syntax score greater than 31 as the best cut off value predictive ofno-reflow phenomenon with sensitivity of $95.1 \%$ and specificity of $94.5 \%$ and area under ROC curve $97.3 \%$, p value $<0.001$.((Figure 1)). 
Table (4): Independent predictors of No-reflow

\begin{tabular}{|c|c|c|c|c|}
\hline & \multirow{2}{*}{$\begin{array}{c}\text { Odds } \\
\text { ratio }\end{array}$} & \multicolumn{2}{|c|}{ 95\% C.I. } & \multirow{2}{*}{ P value } \\
\cline { 3 - 4 } & & Lower & Upper & \\
\hline Age & 8.884 & 2.145 & 36.800 & $\mathbf{0 . 0 0 3}$ \\
\hline Time to reperfusion & 13.844 & 3.214 & 59.636 & $<\mathbf{0 . 0 0 1}$ \\
\hline Target lesion length & 8.637 & 1.975 & 37.768 & $\mathbf{0 . 0 0 4}$ \\
\hline Initial TIMIflow & 20.861 & 1.739 & 250.290 & $\mathbf{0 . 0 1 7}$ \\
\hline Initial TMPG & 0.851 & 0.036 & 20.116 & 0.920 \\
\hline Thrombus burden & 3.262 & 0.769 & 13.831 & 0.109 \\
\hline Killip class III / IV & 1.468 & 0.210 & 10.249 & 0.698 \\
\hline EF \% & 2.476 & 0.191 & 32.087 & 0.488 \\
\hline PVD & 1.870 & 0.540 & 3.212 & 0.755 \\
\hline
\end{tabular}

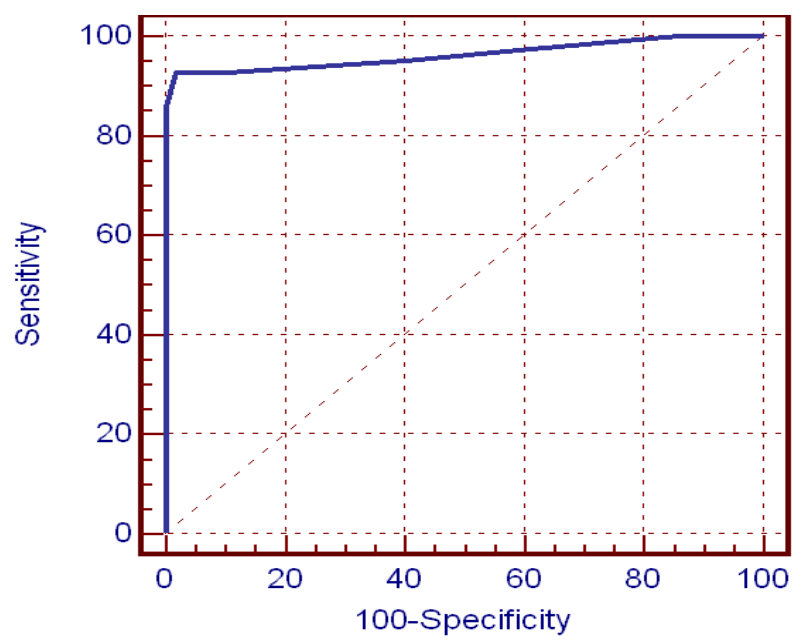

Source of the curve

Syntax score

Figure (1): The ROC curve analysis to show the best cutoff value of the SYNTAX score that identifies No-reflow.

\section{DISCUSSION}

The present study evaluated the ability of the SS to predict no-reflow phenomenon in patients with acute myocardial infarctiontreated with percutaneous coronary intervention.

The main findings of the present study were as follows. (a) no-reflow phenomenon occurred in $26 \%$ of patients with STEMI treated with PPCI and NSTEMI patients treated with early invasive strategy. (b) Patients withno-reflow were older, had higher prevalence of PVD, higher peak CKMB levels, higher RBS levels on admission, higher level of killip class (III / IV), and they tended to have more worse LV contractility, had a longer symptom to balloon time for STEMI patient,.No-reflow was more common in patients who had a low $(\leq 1)$ initial TIMI flow and a low initial TMP grade $(\leq 1)$, also for patients with high thrombus burden, and those who had longer lesions $>$ $20 \mathrm{~mm}$ (c) The multivariable logistic regression model identified The SYNTAX score, the time to reperfusion $>6$ hours for STEMI patients, age $>60$ years, low initial TIMI flow $(\leq 1)$, a long target lesion as independent predictors of no-reflow. (d) ROC curve analysis revealed that SS greater than 31 had a sensitivity of $95.1 \%$, specificity of $94.9 \%$, and area under the ROC curve of $97.3 \%$ for predicting noreflow.
In our study, the incidence of no-reflow was $31 \%$, although the incidence of no-reflow during PCI has ranged from $12 \%$ to $25 \%$ in some studies that have used the criterion of TIMI flow grade $\leq 2^{(12-13)}$. In some studies, the incidence has been $29 \%$ using the TIMI myocardial perfusion grade ${ }^{(\mathbf{2 1})}$, and $34 \%$ to $39 \%$ using myocardial contrast echocardiography ${ }^{(14-15)}$.

We found that the high SxS group compared to the low SxS had more no reflow $(37.4 \pm 3.8$ Vs $22.2 \pm$ 5.8 with $\mathrm{P}$ value $<0.001$ ), patients with a high $\mathrm{SxS}$ had a more complex anatomy of coronary arteries including multivessel disease, diffiuse disease, bifurcation lesions, chronic total occlusion and left main disease. These features could make the procedure of pPCI more difficult and complicated. Therefore, myocardial no-refow could be expected in patients with complex coronary anatomy. Magro et al..$^{(16)}$ found similar results in their study. They examined 669 patients admitted with STEMI and found that postPCI no-reflow rate of patients with high $\mathrm{SxS}(\mathrm{SxS}>$ 16) was significantly high.This was in concordance with Sahin $\boldsymbol{e t} \boldsymbol{a l} .{ }^{(17)}$ and Kammler $\boldsymbol{e t} \boldsymbol{a l} .{ }^{(18)}$ who all found that high SxS group had more no-reflow. 
Iwakura et al. ${ }^{\left({ }^{(19)}\right.}$ found no-reflow phenomenon in 79 of 199 patients $(39.6 \%)$ using myocardial contrast echocardiography 15 minutes after percutaneous coronary intervention and reported a statistically significant higher agein the no reflow group compared to the optimal flow group (64 years vs. 58 years respectively, $\mathrm{p}$-value $=0.003$ ).

Also, Cafri et al. ${ }^{(\mathbf{2 0 )})}$ and Oduncu et al. ${ }^{(21)}$ showed a significantly higher age in the no reflow group compared to the optimal flow group Furthermore, delayed reperfusion can result in an older, more organized intracoronary thrombus which may increase the risk of distal embolization during $\mathrm{pPCI}$ and reduce the likelihood of achieving TIMI flow grade 3 after the procedure. Our study showed a statically significant longer pain to door time in the no reflow group compared to the optimal flow group (7.8 \pm 2.4 vs. $3.4 \pm 1.6$, p-value <.001). This goes in concordance with McNamara et $\boldsymbol{a l} .{ }^{(22)}$ and Ndrepepa et $a .^{(23)}$, where they all showed that the time from onset ofchest pain to the emergency room arrival was significantly higher in the no reflow group compared to the optimal flow group.

Delayed reperfusion (a long duration from onset to reperfusion) is related to no-reflow. Our study showed that patients with a long duration before reperfusion $(>6 \mathrm{~h})$ had a significantly greater thrombus burden and an increase in the no-reflow rate than patients with a short duration of reperfusion.

This is in concordance with Yip et al. ${ }^{(24)}$ who demonstrated that in patients with AMI who had a high thrombus burden, the rate of no-reflow was lower than in those with reperfusion in less than $4 \mathrm{~h}$. This indicates the possible correlation of a thrombus burden with the duration of reperfusion. Tanaka et al. ${ }^{(25)}$ used IVUS to examine plaque burden and identified that a higher lipid content in the inner plaque core and the width of the external elastic membrane were independent markers for the no-reflow phenomenon.

Systolic function of the left ventricle after AMI is one of the most important predictors of long-term outcomes ${ }^{(26)}$. In our study, patients with high SxS had lower EF because more no-reflow developed in patients with high $\mathrm{SxS}$, inadequate reperfusion occurred in the myocardium despite patent IRA.

Our study revealed that Patients with lesions that were longer than $\mathbf{2 0}$ mmwere more likely to develop no-reflow after primary PCI than those with lesions that were shorter than $20 \mathrm{~mm}$ in size. Large vessels are able to contain large amounts of plaque lipid or thrombi. The larger the lesioned vessels, the slower the flow velocity. The longer the target lesion, the larger the amount of thrombus and plaque burden. This would explain the high risk for slow/no-reflow that was observed in these patients after primary $\mathrm{PCI}^{(27)}$.

This goes with what Kirmaet al. ${ }^{(28)}$ reportedin a series of 382 consecutive patients with AMI who underwent primary PCI who showed that advanced age (> 60 years), delayed reperfusion $(\geq 4 \mathrm{~h})$, low $(\leq \mathbf{1})$ TIMI flow prior to PCI, cut-off type total occlusion, high thrombus burden according to baseline angiography, the presence of a long target lesion $(>13.5 \mathrm{~mm})$ and large vessel diameter all correlated with no-reflow.

Among the multiple strategies postulated to prevent and treat this phenomenon, direct stenting without predilation have demonstrated a net clinical benefit. We found a statistically significant higher proportion of patientswho were subjected to direct stenting in the optimalflow group compared to the no reflow group (60.8\%vs. 38.8\% respectively with pvalue $<0.001$ ). Wefound no significant difference between no reflowgroup and optimal flow group as regards the useof thrombus aspiration as an adjunctive to pPCI. Probably, a better selection of patients before theprocedure and an earlier implementation of thepresent or other promising strategies, as the combination of thrombus aspiration and intracoronary infusion of IIb-IIIa Stone $\boldsymbol{e t}$ al. ${ }^{(\mathbf{2 9 )}}$ might represent a greater benefit.

\section{Limitation of this study:}

The present study has several limitations. Firstly, part of the study was retrospective performedat a single center which could have led to bias andmodified the outcomes. The diagnosis of no-reflowwas made considering only the epicardial flow and that we didn't analyze the microvascular function and no-reflow using myocardial contrast echocardiography or nuclear scintigraphy.

Despite these considerations, we think that these results are convincing and highly significant, and should be confirmedby prospective studies.

\section{CONCLUSIONS}

Syntax score (Sxs) is an independent predictor of no reflow phenomenonand thus can be used to stratify AMI patients into low or high risk for angiographic noreflow.

Predictors of no reflow after primary PCI using univariable analysis showed 9 variables (age, time to reperfusion,target lesion length, initial TIMI flow , initial TMPG , thrombus burden, Killip class III / IV , EF , PVD. ).

The confirmation of these findingsin prospective studies might allow the implementation of strategies to prevent this phenomenonand eventually improve the long term clinicaloutcomes.

\section{REFERENCES}

1. Ito H, Tomooka T, Sakai $\mathbf{N}$ et al. (1992): Lack of myocardial perfusion immediately after successful thrombolysis. A predictor of poor recovery of left ventricular function in anterior myocardial infarction. Circulation, 85:1699-705.

2. Giampaolo N, Rocco AM, Leonardo C et al. (2014): Morphological-biohumoral correlations in acute 
coronary syndromes: pathogenetic implications. Int $\mathbf{J}$ Cardiol., 171(3):463-6.

3. Kloner RA, Ganote CE, Jennings RB et al. (1974): The 'no-reflow' phenomenon after temporary coronary occlusion in the dog. J Clin Invest., 54: 1496-508.

4. Kotani J, Nanto S, Mintz GS et al.(2002): Plaque gruel of atheromatous coronary lesion may contribute to the no-reflow phenomenon in patients with acute coronary syndrome. Circulation, 106:1672-1677.

5. Wong DT, Puri R, Richardson JD et al. (2013): Myocardial 'no-reflow'--diagnosis, pathophysiology and treatment Int J Cardiol., 167(5):1798-806.

6. Garg S, Serruys PW, Silber S et al. (2011): The prognostic utility of the SYNTAX score on 1-year outcomes after revascularization with zotarolimus- and everolimus-eluting stents: a substudy of the RESOLUTE All Comers Trial. JACC Cardiovasc Interv., 4:432-441.

7. Kristian T, Joseph SA, Allan SJ et al.(2012): The Writing Group on behalf of the Joint ESC/ACCF/AHA/WHF Task Force for the Universal Definition of Myocardial Infarction. European Heart Journal, 33: 2551-2567

8. Georgios S, Marie-Angèle M, Arie PK et al.(2005): The SYNTAX Score: an angiographic tool grading the complexity of coronary artery disease. EuroInterv, $1: 219-227$

9. TIMI Study Group (2009): Definitions used in TIMI trials. Available at: http: // www.timi.org.

10. van't Hof AW, Liem A, Suryapranata H et al. (1998):Zwolle Myocardial Infarction Study Group. Angiographic assessment of myocardial reperfusion in patients treated with primary angioplasty for acute myocardial infarction: myocardial blush grade. Circulation, 97:2302-6.

11. Khalill R, Lei $\mathbf{H}$ and Chang $\mathbf{J}$ (2008): The diagnosis and treatment of the no-reflow phenomenon in patients with myocardial infarction undergoing percutaneous coronary intervention.ExpClinCardiol., 13(3): 121-128.

12. Morishima I, Sone T, Okumura K et al. (2000): Angiographic no-reflow phenomenon as a predictor of adverse long-term outcome in patients treated with percutaneous transluminal coronary angioplasty for first acute myocardial infarction. J Am Coll Cardiol., 36: 1202-1209.

13. Piana RN, Paik GY, Moscucci M et al. (1994): Incidence and treatment of 'no-reflow' after percutaneous coronary intervention. Circulation, 89: 2514-2518.

14. Dibra A, Mehilli J, Dirschinger $J$ et al. (2003): Thrombolysis in myocardial infarction myocardial perfusion grade in angiography correlates with myocardial salvage in patients with acute myocardial infarction treated with stenting or thrombolysis. J Am Coll Cardiol., 41: 925-929.

15. Ito H, Okamura A, Iwakura K et al. (1996): Myocardial perfusion patterns related to thrombolysis in myocardial infarction perfusion grades after coronary angioplasty in patients with acute anterior wall myocardial infarction. Circulation, 93: 1993-1999.

16. Magro M, Nauta ST, Simsek C et al. (2012): Usefulness of the SYNTAX score to predict "no reflow" in patients treated with primary percutaneous coronary intervention for ST-segment elevation myocardial infarction. Am J Cardiol., 109: 601-606.

17. Sahin DY, Gür M, Elbasan Z et al. (2013): Mean platelet volume and extent and complexity of coronary artery disease in diabetic and nondiabetic patients with ST elevation myocardial infarction. Angiology, 64: 505511.

18. Kammler J, Kypta A, Hofmann R et al. (2009): TIMI 3 flow after primary angioplasty is an important predictor for outcome in patients with acute myocardial infarction. Clin Res Cardiol., 98(3): 165-70.

19. Iwakura K, Ito H, Kawano $\mathrm{S}$ et al. (2001): Predictive factors for development of the no-reflow phenomenon in patients with reperfused anterior wall acute myocardial infarction. J Am Coll Cardiol., 38:472-7.

20. Cafri C, Zahger D, Merkin M et al. (2013): Efficacy of the radial approach for the performance of primary PCI for STEMI. J Invasive Cardiol., 25(3): 150-3.

21. Oduncu V, Tanalp AC, Erkol A et al. (2011): Impact of chronic pre-treatment of statins on the level of systemic inflammation and myocardial perfusion in patients undergoing primary angioplasty. Am J Cardiol., 107(2): 179-85.

22. McNamara RL,Tanalp AC, Erkol A et al. (2006): Effect of door-to-balloon time on mortality in patients with ST-segment elevation myocardial infarction. J Am Coll Cardiol., 47 (11): 2180- 6.

23. Ndrepepa G, Tiroch K, Keta D et al. (2010): Predictive factors and impact of no reflow after primary percutaneous coronary intervention in patients with acute myocardial infarction. Circ Cardiovasc Interv., 3(1): 27-33.

24. Yip HK, Chen MC and Chang HW (2002): Angiographic morphologic features of infarct-related arteries and timely reperfusion in acute myocardial infarction: predictors of slow-flow and no-flow. Chest, 122:1322-1332.

25. Tanaka A, Kawarabayashi T and Nishibori Y (2002): No-reflow phenomenon and lesion morphology in patients with acute myocardial infarction. Circulation, 105:2148-2152.

26. Vasan RS, Larson MG, Benjamin EJ et al. (1999): Congestive heart failure in subjects with normal versus reduced left ventricular fraction: prevalence and mortality in a population-based cohort. AmCollCardiol., 33: 1948-1955.

27. Bae JH, Kwon TG, Hyun DWet al. (2008): Predictors of slow flow during primary percutaneous coronary intervention:An intravascular ultrasound virtual histology study. Heart, 94:1559-1564

28. Kirma C, Izgi A andDundar C (2008): Clinical and procedural predictors of no-reflow phenomenon after primary percutaneous coronary interventions: experience at a single center. Circ J., 72:716-721.

29. Stone GW, Maehara A, Witzenbichler B et al. (2012): Intracoronary abciximab and aspiration thrombectomy in patients with large anterior myocardial infarction: the infuse-AMI randomized trial. doi: 10.1001/jama.2012.421. http://doi.org/k6h, 2012. 